\title{
A Fast and Efficient Lossless Compression Technique for Greyscale Images
}

\author{
T. Kavitha, K. Jaya Sankar
}

\begin{abstract}
The growth of cloud based remote healthcare and diagnosis services has resulted, Medical Service Providers (MSP) to share diagnositic data across diverse environement. This medical data are accessed across diverse platforms, such as, mobile and web services which needs huge memory for storage. Compression technique helps to address and solve storage requirements and provides for sharing medical data over transmission medium. Loss of data is not acceptable for medical image processing. As a result, this work considers lossless compression for medical in particular and in general any greyscale images. Modified Huffman encoding (MH) is one of the widely used technique for achieving lossless compression. However, due to longer bit length of codewords the existing Modified Huffman (MH) encoding technique is not efficient for medical imaging processing. Firstly, this work presents Modified Refined Huffman (MRH) for performing compression of greyscale and binary images by using diagonal scanning method. Secondly, to minimize the computing time parallel encoding method is used. Experiments are conducted for wide variety of images and performance is evaluated in terms of Compression Ratio, Computation Time and Memory Utilization. The proposed MRH achieves significant performance improvement in terms of Compression Ratio, Computation Time and Memory Usage over its state-of-the-art techniques, such as, LZW, CCITT G4, JBIG2 and Levenberg-Marquardt (LM) Neural Network algorithm. The overall results achieved show the applicability of MRH for different application services.
\end{abstract}

Keywords: Cloud computing, codeword, Compression, Huffman, Variable Length Code.

\section{INTRODUCTION}

The rapid growth of the multimedia industry and increasing demand of multimedia application services, encourage the researcher to work on promising technologies to utilize storage and communication capacity efficiently. Further, the future multimedia application requires real-time service delivery. Specially, in the field of Healthcare and diagnosis. The emergence of cloud computing in the field of healthcare and diagnosis provision numerous aids such as computing resource, storage resources and real-time access to medical data. Provisioning admittance to these medical (diagnostic) image on cloud platform will aid in reducing duplication cost, distribution cost, and the cost of storing diagnostic data. The stored medical data on cloud platform are shared among service providers, users and are accessed by heterogeneous platforms such as mobile and web.

T. Kavitha is working as Associate professor in the Department of E.C.E, M.V.S.R Engineering College,Nadergul, Hyderabad, Telangana, India 500036. Phone: 07569110111, e-mail: tkavitha ece@mvsrec.edu.in.

K. Jaya Sankar is working as Professor and Principal in Mahatma Gandhi Institute of Technology, Hyderabad, Telangana, India, e-mail:

kottareddyjs@gmail.com
The need of interoperability among platforms has initiated the adoption of DICOM standard. Further, the storage and bandwidth constraint may perquisite good encoding for healthcare related diagnostic data.

The cloud based healthcare system prerequisite the need for good compression technique, which is lossless in nature. The challenges involved to access medical images through smart devices were highlighted in [1]. Generally, the medical images are wider than the images of Computed Radiography (CR), Magnetic Resonance (MR), Computed Tomography (CT),

Ultrasound (US), and Positron Emission Tomography (PET) CT (PET-CT) [2]. Medical diagnosis of a patient involves a set of diagnostic feature data. As a result, large size of data is produced. For example, a complete body PET may involve around six hundreds diagnostic data per feature, where the size of each set is 1.2 giga bytes. For performing analysis there are four sets. Thus, the total size will be of 4.8GB which has to be stored at superior quality. Therefore, good encoding method for medical images will aids in reducing cost and improving access on heterogeneous devices, such as, smart phones, tablets etc.

Image compression plays an important role in the field of multimedia image processing. The image compression technique is widely used across different fields such as satellite imaging and medical imaging etc. The image compression aids in reducing the image size, so that the encoded images can be transmitted over the communication channel between source and destination in minimal time. Compressing images helps in storing more images in storage devices [3]. To achieve lossless compression, Huffman algorithm is used which is a wellknown minimum redundancy code when compared to other state-of-the-art techniques [4]. The Huffman coding technique has been effectively used in image, text, video encoding and real-time video conferencing system such as JPEG, MPEG-4, and H.263 etc.[5].The Huffman coding method gathers symbols from source images/text and computes probabilities of all the symbols and sorts the symbols based on its probability. Then, a binary tree is formed by combining two symbols at a time from the lowest probability to the highest probability symbols. Besides, allocating one to the right node and zero to the left node staring from the root of the tree. To obtain a Huffman codeword for a specific symbol, all ones and zeros collected from the root to that specific node are to be considered in the same order [6].

Recently, a number of compression techniques have been presented for medical image processing [7]. In [7], carried out performance evaluation for both lossless and lossy for healthcare multimedia data and outcome shows reduction in spectrum usage, cost of communication storage overhead 
cost. Nonetheless, it incurs higher computation time. The computation time minimization problem of [7] is addressed by [8] HEVC which adopts HEVC encoding standards. However, to achieve compression without any loss of information considering coding tree unit [9] requires parallel computing (PC) framework [10]. In [12], presented VariableLength Code based encoding methods and [13] presented an hybrid encoding method, both [12] and [13] have adopted Huffman codeword or encoding method [14] for minimizing average bit size of the Huffman codewords, to provision enhanced encoding without losing any information considering wireless transmission medium and to overcome limitation of fixed length coding [11].

To further, improve compression performance and support compression for grayscale images [15] presented Modified Modified READ (MMR) which is a modification of Huffman algorithm. In [16] presented a multi-layer neural network based compression technique for performing compression on Bi-level images. The neural network takes foreground and background feature classes as inputs for training and in the reconstruction phase, the pixels addresses are fed to the network inputs and the network output is obtained. They attained significant performance over their predecessor [15] in terms of compression ratio. However, the model need to be retrained for new type of object resulting in NP-Hard problems. As a result, it induces computational complexity.

In this work, researcher focuses on designing a scalable lossless image compression with minimal computation overhead/complexity. Despite the wide adoption of lossy coding techniques for applications, such as, art archiving, technical drawing, and medical imaging, the lossless compression is more appropriate for achieving highly reliable encoding without loss of data. Therefore, most of the image compression techniques presented are lossless compression [10-16]. The important performance evaluation parameters for these techniques is Compression Ratio, since its primary objective it to minimize the bandwidth and storage space. For future applications dynamic requirement of memory and computation complexity should also need to be considered as an important performance parameters. In various multimedia applications, as in high-definition television and internet, high resolution images are usually desired by end-users. However, images which are huge in size require more computations, which results in coding latency at terminals. Hence, a scalable and efficient compression technique with minimal computation is required to minimize the latency and fine-tune the image resolution according to the user requirement and channel capacity. Further, when implementing compression standard in embedded systems, as in smart phones and digital cameras, the low complexity coding standards are preferred to minimize the battery power consumption and computation time. In such cases, use of reduced length of codewords is the required solution for both encoding and decoding.

\section{The contribution of the research work is presented below:}

- This paper presented a fast and efficient encoding (compression) technique named Modified Refined Huffman (MRH).

- To the best of our knowledge no prior work has considered diagonal scanning encoding method.
- Experiments are conducted for both Greyscale and Bilevel Images; performance is evaluated in terms of compression efficiency (CE) and overall processing time (OPT).

- The proposed MRH attains good enhancement in terms of CE and OPT over its state-of-the-art techniques mentioned in [15], [16] and [17].

The manuscript is arranged as follows. The survey of existing compression techniques is discussed in section II. The proposed Modified Refined Huffman compression method is presented in section III. In Section IV, the results obtained are discussed. Last section presents conclusion and future research direction.

\section{LITERATURE SURVEY}

Several methodologies has been presented in recent times to improve compression performance, reduce computation time and utilize memory efficiently for both Bi-level and greyscale images which are surveyed here. In [19], Visual Sensor Nodes (VSN) obtain images from different areas of field and perform processing on images and the results are transmitted using embedded wireless transceivers. These, VSN all together forms a wireless Sensor Network. However, VSN introduces high amount of redundancy, the compression efficiency is dependent on the algorithm. Here, performance of compression rate is increased by detecting ROI (Region of Interest) for lossless coding standards which include various shapes, locations and different objects. Three different compression techniques namely Joint Bi-level Image Group (JBIG2), CCITT Group4 and Gzip are used as coding standards for discussion. The compression performance is best in CCITT Group4 technique with shows low computational complexity. This paper, focuses on the enhancement of compression efficiency for greyscale and binary images. However, CCITT Group 4 introduces increase in size of compressed files compared to other JBIG2 and Gzip coders.

In last few decades, in the field of data compression arithmetic and Huffman coding methods proved as most efficient entropy encoders. Both encoding methods are different from each other. One is symbol wise coding while other is continuous coding. Optimality of the encoding technique depends on how best the encoder reduces redundancies present in the data. The probability of source symbols is an essential information for any statistical (entropy) coder. However, the above coding methods are very timeconsuming. Instead of arithmetic coding, a simple and nonprobabilistic method for bi-level compression is suggested in [20] namely Context-based Sorting and Interpolative coding, version $1 \&$ version 2 (CSI-1 and CSI- 2). A standard context model in association with interpolative encoding provide high compression rate than JBIG and JBIG2 standard coding and processing time required is very less. This process provides better compression rate. The experimental results using CSI-1 and CSI-2 are compared with existing techniques like JBIG and JBIG2. Using CSI-1 technique the compression gain is comparable but computational complexity is also very high. 
In [10], authors set up an effective encoding method to enhance the processing time of encoding image/text. They exhibited a wavelet-based encoding strategy by utilizing parallelization in system. They adopted GPU (graphical processing unit) platform for attaining fine-grained parallelization using Biplane compression technique. Efficient thread and memory optimization technique is modelled for mapping data to attain superior association between communicating intermediate threads. Performance evaluation is conducted by carrying experiment on images with high resolution. The result obtained shows less energy utilization with better speedup.

In [27], author developed a model to construct a dictionary to represent sparse multimedia data. They adopted dictionary based learning technique to build collaborative dictionaries for sparse multimedia data. They presented boosting based dictionaries which resulted in obtaining better sparse representation. Two optimization models are presented that improve generalization characteristics of trained dictionaries. Based on this dictionary, they developed a new compression technique. The outcome of the experiments conducted and outcome showed better performance than JPEG-2000 [33] in terms of Peak-signal-to-noise ratio (PSNR) and Bit-error rate (BER).

In [28], established an efficient compression technique to improve the speed up of compression, they presented a wavelet-based image coding method by adopting parallelization technique. This model is parallelized with GPU to achieve fine grained parallelism by adopting Bit plane coding. The model also presented a smart memory management and thread to data mapping mechanism for better communication among inters threads. The outcome of the experiments conducted for high resolution images showed that it achieves better speedups and it also consume less power or energy.

In [29], constructed a codebook and presented vector quantization technique to determine the quality of compressed data. One of the widely used codebook designs; Linde-BuzoGray (LBG) [31], [32] tends to suffer from local minima problem and gets trapped to local minima for initial codebook generation. To address this, they designed a high quality initial codebook design model. The model divides the training vector into groups by utilizing variance and means characteristics parameter of training vectors. Then the initial codebook is generated by choosing code word from each group. Experiments are conducted to evaluate the performance of their initial codebook generation over the existing technique. It showed better performance in terms of PSNR.

In [30], author presented a binary arithmetic encoding method with fixed length codewords based on variable size sliding window method. The renormalization overhead issues arising due to longer coding size of Apriori unidentified coding size is addressed in this work. Using varying coding length/size and sliding window the probabilities are estimated to identify good correctness codes. Thus, their model is adaptive in nature. Results are obtained in terms of coding rate and processing time which showed that their approach attained superior outcome when compared with M coding and MQ coding technique.
In [11], presented binary arithmetic encoding method considering context awareness with fixed code size. The renormalization overhead issues arising due to longer coding size of apriori unidentified coding size is addressed in this work. Using variable length coding and sliding window mechanism, the probabilities are estimated to identify proper codes. Thus, their model is adaptive in nature. Results are obtained in terms of coding rate and processing time which shows their approach attains superior outcome when compared with $\mathrm{M}$ coding and MQ coding technique.

In [21], a binary image compression technique is presented for FPGA implementation. Digital image processing is widely utilized in many applications namely Medical, Machine Learning and Remote Sensing etc. However, these applications need a large amount of data and transmission cost to represent these images. There are many existing techniques available to perform compression for various purposes, either lossy or lossless. Here, a study is presented to identify the behavior of various techniques for different image sets. All the techniques are compared with each other in terms of compression efficiency, time and ratio. The compression algorithm is presented with FPGA implementation workflow. This paper helps in the selection of best compression technique, six types of compression algorithms which are utilized namely Packbits, Lempel- Ziv-Welch (LZW), LZ77, Zip (Deflate), JBIG2 and CCITT Group $3 \& 4$. Among these entire algorithms CCITT G4 algorithm is one of the best algorithm. However, computational complexity is very high.

In [26], author has proposed LSB algorithm for image security. The cover images are embedded at various LSB positions by computing the histogram of an image. A novel embedding scheme is presented to reduce the attacks on histogram. The experimental results shows the PSNR of reconstructed images is better and it is efficient for embedding attacks.

In [16], author proposed a neural network based Levenberg-Marquardt LM back propagation algorithm for binary images using a multi-layer perceptron network. The algorithm is a lossy and the analysis shows higher compression ratios are achieved in comparison with the other state-of-theart compression techniques like LZW, Deflate, Packbits ,CCITT G3 \& G4 and as well as JBIG2.

From the extensive literature survey carried out here, shows need for new lossless compression technique that brings a good trade-off between compression and computation performance for various kind of medical and other greyscale images. The author has proposed a dictionary of optimized prefix free Huffman Code words [17] [22] namely Refined Huffman (RH) that proved to be more efficient than the existing Modified Huffman (MH). The compression ratios obtained for binary and halftone images is presented in [17] [22]. In this paper the research work is extended to greyscale images and author demonstrated that the proposed Modified Refined Huffman (MRH) proved to achieve better compression ratios then many of the other state-art-of-art algorithms. 
In the next section, the proposed Modified Refined Huffman algorithm to compress medical and general greyscale images is presented and the result analysis is presented in the result section to prove the claim that the proposed MRH gives better performance.

\section{PROPOSED MODIFIED REFINED HUFFMAN (MRH) FOR COMPRESSING GREYSCALE IMAGES}

Here, we present a fast and efficient image compression technique for encoding greyscale images using Modified Refined Huffman (MRH) technique. To achieve fast and efficient compression for grey scale images the following steps are considered.

- The author has presented Refined Huffman (RH) which is a 1-D coding $[17,22]$ to minimize the number of codewords, length of codewords and average length of codewords.

- The author proposes 2-D coding in this paper, Modified Refined Huffman namely MRH to minimize the computation time and achieve better compression by adopting diagonal scanning method in both directions in parallel; it is a 2-D coding technique.

Firstly, a Refined Huffman (RH) 1-D coding is explained in brief presented by author in [17, 22] how number of codewords, length of codewords and average length of codewords is reduced. Secondly, the author proposes a 2-D coding ,Modified Refined Huffman namely MRH extension to $\mathrm{RH}$ to minimize computation time and achieve better compression by adopting diagonal encoding in parallel fashion for greyscale and binary images; it is a 2-D coding technique. MRH proves to be better than Modified Modified Read (MMR) and RH, MMR is explained briefly in the next paragraphs.

\section{A. Refined Huffman}

The binary image contains runs of white and black pixels which make run length encoding (RLE) efficient for minimizing these run lengths. The RLE compressed run lengths are then combined with Huffman coding. Combining RLE with Huffman coding is known as Modified Huffman (CCITT G-3) is a 1-D coding [23] and run lengths are encoded line by line. To encode these Run lengths, the total number of prefix free codes are 183 in number exceedingly large and require large memory space. Maximum length of codeword is13 and there are quite a good number of codes with length 12. It takes large coding and decoding times because of longer lengths of codes. As a result compression achieved is very less [14]. It also incurs computation overhead due to longer length of codewords.

To address the memory and computation overhead, Refinement of Huffman namely, RH is presented in [17], [22], author proposed new Look-up table. The work presented in [17], [22] considers minimizing number of codewords to $\mathbf{7 1}$ from 183 and average length of codeword is reduced to 7.7046bits/symbol from 8.6270 bits/sample for Modified Huffman (MH), without out any data loss. The developed codewords for run lengths of white and black follows the series of Fibonacci numbers.

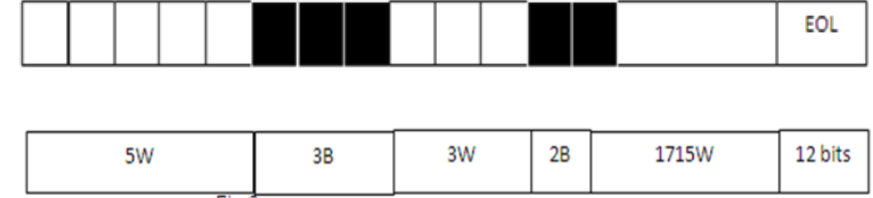

\begin{tabular}{|l|l|l|l|l|c|}
\hline 1110 & 10 & 1000 & 11 & 01100001010100 & 000000000001 \\
\hline
\end{tabular}

Fig.1. Refined Huffman Encoding Example.

An example to prove that the proposed RH [17[, [22] reduces the number of bits for encoding is shown in Fig.1.Consider a row of an image consisting of 1728 white and black pixels. The run lengths are also shown in the figure and last figure of Fig. 1 shows the encoded run lengths using RH dictionary [17],[22] and it can be seen that total of 1728 pixels are encoded equal to 35 .If we encode the same line with Modified Huffman (MH ) it will be equal to 45 bits. So, using $\mathrm{RH}$ for one line of coding 10 bits are saved. In this paper, the work is extended to greyscale images and anew scanning technique is also proposed which is explained in the following sections.

Grey scale image is a combination of binary bit planes. Each bit plane consists of runs of black and white pixels, so here Refined Huffman (RH) can be easily applied. As RH is a 1-D coding and combines Run Length Encoding (RLE) and Huffman coding. The maximum size of any codeword in Modified Huffman (MH) is 13, whereas, maximum size of proposed RH is 12 [17]. The proposed codewords/Look-up table is shown in [17]. Due to longer size of the codewords, traversing $\mathrm{MH}$ binary tree is far higher than traversing $\mathrm{RH}$ binary tree. Also, encoding/decoding time and memory utilization is much lower for $\mathrm{RH}$ due to refinement of codewords proved in [17], [22].

Though, RH has attained good compression and reduced the computation time it is not efficient to compress medical images. Since, medical images are composed of grey areas and neighboring pixels are highly co-related. As a result $\mathrm{MH}$ and $\mathrm{RH}$ performs poorly. Since, MH and RH does not exploit the correlation between pixels i.e 2-D. Therefore, for achieving better compression and computation efficiency, we further present a refinement of RH, namely, MRH (Modified Refined Huffman). MRH is a 2-D technique and exploits correlation between pixels and as well as similarities between successive lines which outperforms Modified Modified read (MMR), a 2D coding technique.

B. Modified Modified READ (MMR) and Proposed Modified Refined Huffman (MRH) Coding:

The MRH involves the following steps, image subdivision, intensity computation, pattern determination. As two adjacent scan lines of a greyscale image are highly correlated. Therefore, the run length of encoding line can be coded with respect to the run lengths of the previous encoding line. In MRH coding, before encoding a run length, it first establishes 
five reference pixels on present and previous scan line, which are represented by $a_{0}, a_{1}, a_{2}, b_{1}$ and $b_{2}$ respectively.

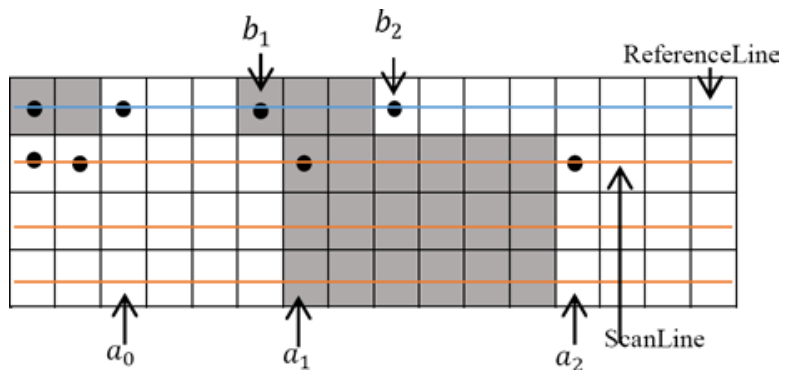

Fig. 2: Existing MMR: Two rows of an image, the transition pixels are marked with a dot.

The coding modes used are pass, vertical and horizontal shown in Fig.3.Detailed explanation can be found in [24].

\section{Definition of picture elements and coding modes:}

Referring to Fig. 2 and 3. The initial picture elements and the changing picture elements are defined as follows, $a_{0}$ is the starting picture element on the coding line, which becomes the reference picture element, $a_{1}$ is the changing picture element on the coding line, right next to $a_{0}, a_{2}$ is the changing picture element on coding line, right next to $a_{1}, b_{1}$ is the first changing picture element, whose color is opposite to $a_{0}$, which occurs on the previous line (Reference) after the picture element toward right of $a_{0}$ and $b_{2}$ is the first changing picture element toward right of $b_{1}$ on the reference line.

For our case study, let us consider that, the second row is to be encoded. Then, the pixels on the reference line are encoded with respect to second row. Thus, the identification and definition of pixel elements is shown in Fig.2 and 3, respectively. The encoder (Transmitter) and decoder (Receiver) has prior information of position of $a_{0}, b_{1}$ and $b_{2}$. However, the information of position of $a_{1}$ and $a_{2}$ are known only to the transmitter.Based on the relative position of these pixels the selection of modes can be defined as shown in Fig.3. If the run lengths on present and reference line are similar then the distance among $a_{0}$ and $b_{1}$ would probably be very less when compared with the distance among $a_{0}$ and $a_{1}$.Therefore, the present length can be identified by encoding the distance $\left(a_{1}, b_{2}\right)$ and this mode is called as vertical coding mode.

However, as displacement among $a_{1}$ and $b_{1}$ is higher (i.e. if no existence of identical run on the reference line, then it is ideal to the runs $\left(a_{0}, a_{1}\right)$ and $\left(a_{1}, a_{2}\right)$ to use 1-D run length coding and this type of compression mode is called as horizontal coding mode. Last type of coding is called as pass mode. This mode is performed when b1 and b2 occurs between $\left(a_{0}, a_{1}\right)$. i.e. we process two runs in the preceding line prior to finishing the present run on present line. For this scenario we simply move toward next pixel to be encoded and mark it as new $\left.\mathrm{a}_{0} \overline{(a}_{0}\right)$ which is the pixel on present line that is exactly under $b_{2}$. Prior to transmitting any run sizes, a codeword describing the mode utilized for performing transmission. The information about codewords being utilized to carryout encoding operation to be used is described in [24] and shown in Fig.3.

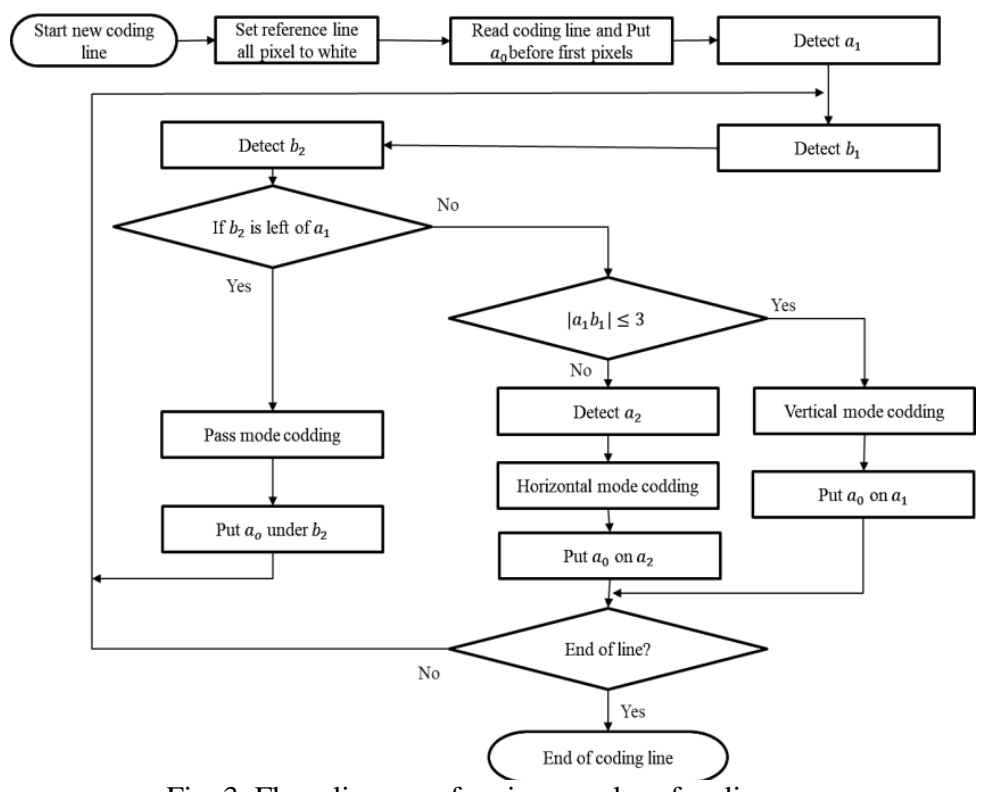

Fig. 3: Flow diagram of various modes of coding

The compression efficiency obtained by 1-D dimensional coding is very limited. Since, it does not take vertical correlation into account i.e. correlation among adjacent scan lines. An efficient way to utilize vertical correlations feature is to perform operation pixels line by line, and use information obtained in preceding encoding lines in order to encode sequence of successive pixels or the current pixels as discussed above.

\section{Proposed MRH:}

This work presents a 2-D dimensional coding method namely Modified Refined Huffman (MRH) that compares current encoding/Scanning line with the previous/reference line. MRH assumes that there is an all-white line, which is considered as first reference line for encoding. It becomes the first reference line and second encoding line is coded with respect to the reference line similar to (Modified Modified READ) MMR. The algorithm of MRH is shown in Fig. 4 and different coding modes such as horizontal, vertical and pass mode are discussed in previous page and shown in Fig.2. If there are no identical runs on the coding line then it is Horizontal coding mode, all such run lengths need to be encoded as 1-D using codewords for white and blacks run lengths. To encode this we have used our developed code words (RH) similar to 1-D coding [17].

In MRH, encoding is done diagonally instead of horizontal as seen in MMR using RH codewords [17] and then the subsequent lines of two diagonal halves are encoded in parallel exploiting the correlation between pixels (2-D), which will aids in reducing the computation time. Thus, MRH is extension of RH work. 


\section{The proposed diagonal scanning method:}

- The image is sub-divided into two diagonal halves and stored into two arrays and both halves are encoded in parallel fashion diagonally as shown in Fig.5 as we proceed from the center diagonal to top and bottom of the image.

- The length of the scanning lines for coding decreases and number of comparisons will reduce as we move from center to top and bottom of the image.

- The correlation between the pixels is exploited when current line is encoded compared with respect to previous line, using modes and definition of picture elements, which will help in reducing the time to encode drastically and as well as results in improved compression ratio.

- Whenever horizontal coding mode occurs, MRH uses RH prefix free coding words proposed by author [17], which will help in achieving improved compression as the average length of the code words is 7.7046bits/symbol compared to $8.6270 \mathrm{bits} / \mathrm{symbol}$ for MH code words.

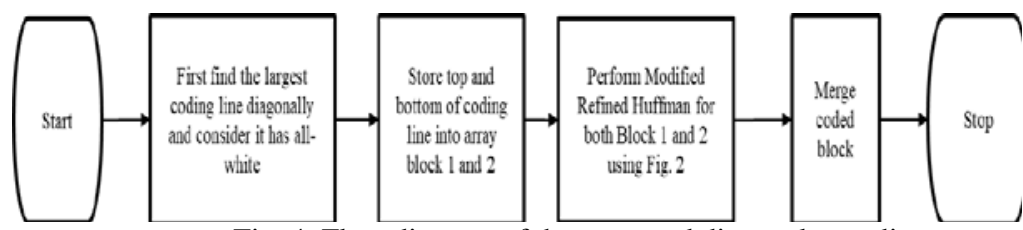

Fig. 4: Flow diagram of the proposed diagonal encoding

\section{Highlights of proposed MRH:}

Comparing Fig.2. and Fig.4., it can be seen that in the existing MMR the size of scan line remains the same where as in the proposed MRH the size of scan line decreases with every successive scan, thus helping in improving compression ratio. For example, let us consider an image $X$ which is of size of $6^{*} 6$ performing encoding on $X$ using MMR needs to scan 36 pixel positions. However, using proposed MRH for encoding image $X$ needs to scan 21 pixel positions. As the proposed model the encoding line size reduces with every successive scan line.

Thus, MRH aids in attaining a better performance than the MMR. Encoding is carried out in both directions in a parallel fashion, thus improving the performance of computing time. Further, the MRH overcomes the limitation of Modified READ (MR) i.e. the MR is dependent on K-factor where every $K^{\text {th }}$ line is encoded using 1-D modified Huffman (MH) encoding. As a result, MR and $\mathrm{MH}$ are not efficient for encoding greyscale images and increase computation time.

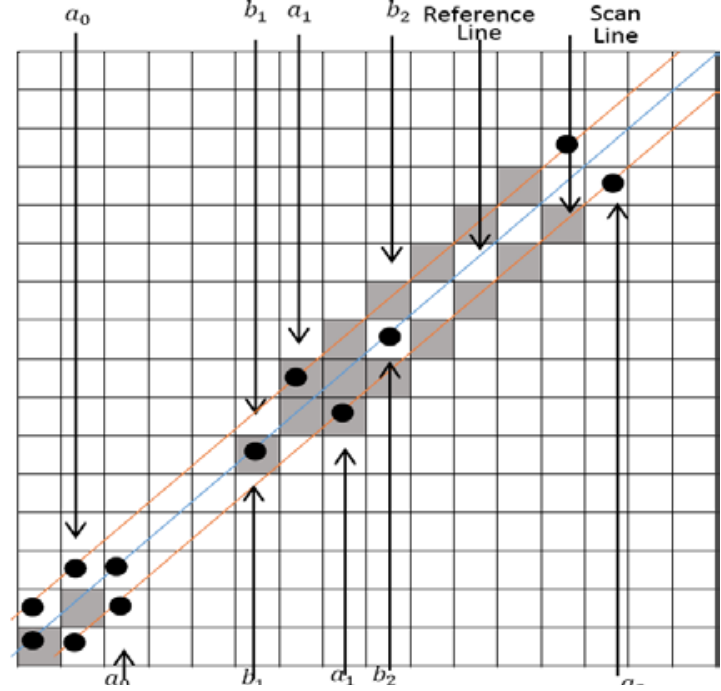

Fig. 5: Proposed Modified Refined Huffman (MRH): Two rows of an image, the transition pixels are marked with a dot.

To overcome, MRH considers diagonal all-white pixels as the first line which is a reference line and every successive line is encoded with respect to reference line and continued in both directions diagonally as shown in Fig.5. This helps in achieving a better compression and reduced computation time for medical images which is experimentally proved in the next section below.

\section{RESULT AND ANALYSIS}

This section describes performance evaluation of MRH over the existing MMR. The algorithm of both MRH and MMR are implemented using C \# 6.0 Microsoft dot net frameworks 4.0, C++ and MATLAB 2016b. The experiments are conducted using windows 10 operating system, Intel I-7 quad core class processor, 16GB RAM and 4 GB dedicated CUDA Graphic Processing Unit. Performance is evaluated in terms of Compression Ratio (CR), Encoding Time (ET), Decoding Time (DT) and Compression Memory Utilized (CMU) for bi-level and grey scale images. We have considered three cases, in first case study we considered bilevel images obtained from [18].For second case bi-level images from [16] and in last case study, we considered greyscale images.

\section{A. Performance evaluation by considering bi-level} document images:

Firstly, this work considers the performance evaluation of bi-level document images for MRH over the existing MMR [15] in terms of CE, EPT, DPT and CMU. The bi-level document images used for analysis are obtained from [18], the first 8 CCITT document images are considered for analysis. All images have same resolution of $200 \mathrm{dpi}$ and same dimension of $(\mathrm{W} * \mathrm{H})$ of $1728 * 2339$. The outcome achieved is tabulated in Tables1 and 2. The results shows that the proposed MRH attained good outcome over the standard MMR method in terms of CE, CMU and computation time considering both EPT and DPT. The image 8 has attained highest compression efficiency of $37.15 \%$ and image 4 has achieved the least CE of $15.33 \%$. An average CE enhancement of $17.29 \%$ is attained by the MRH over the standard MMR technique. The proposed, MRH compression method utilizes 
less memory usage when compared to that of the standard MMR compression method. An average memory usage minimization of $2.07 \%$ is attained for MRH compression technique over the standard MMR compression technique.

Table 1: Performance Evaluation of Compression Efficiency and Compression Memory Utilization

\begin{tabular}{|l|l|l|l|l|}
\hline $\begin{array}{l}\text { Imag } \\
\text { es }\end{array}$ & \multicolumn{2}{|c|}{$\begin{array}{c}\text { Compression } \\
\text { Efficiency }\end{array}$} & \multicolumn{2}{c|}{$\begin{array}{c}\text { Compression } \\
\text { Memory Utilized(bits) }\end{array}$} \\
\hline $\begin{array}{l}\text { Bi- } \\
\text { level }\end{array}$ & $\begin{array}{l}\text { Proposed } \\
\text { MRH }\end{array}$ & $\begin{array}{l}\text { Existing } \\
\text { MMR }\end{array}$ & $\begin{array}{l}\text { Proposed } \\
\text { MRH }\end{array}$ & $\begin{array}{l}\text { Existing } \\
\text { MMR }\end{array}$ \\
\hline 1 & 47.387 & 39.159 & 456626176 & 474959872 \\
\hline 2 & 61.320 & 49.911 & 457134080 & 472010752 \\
\hline 3 & 30.432 & 25.343 & 462970880 & 472938112 \\
\hline 4 & 15.336 & 12.910 & 458014720 & 468649600 \\
\hline 5 & 28.018 & 23.352 & 452780032 & 463550080 \\
\hline 6 & 43.332 & 35.786 & 450174976 & 451240512 \\
\hline 7 & 15.472 & 13.016 & 448634880 & 450471040 \\
\hline 8 & 37.155 & 30.823 & 450093056 & 459847296 \\
\hline AVG & $\mathbf{3 4 . 8 0 7}$ & $\mathbf{2 8 . 7 8 7}$ & $\mathbf{4 5 4 5 5 3 6 0 0}$ & $\mathbf{4 6 4 2 0 8 4 0 8}$ \\
\hline
\end{tabular}

Similarly, the MRH reduces the computation time compared to the existing MMR. The computation time performance is evaluated in term of EPT, DPT, and Overall Processing Time (OPT) and shown in Table 2. An average EPT of 0.0123s (seconds) is attained for presented MRH and 0.088s is attained for standard MMR method. An average EPT minimization of $28.47 \%$ is attained by MRH compression method over the standard MMR compression method. An average DPT of 0.0033 seconds is achieved for $\mathrm{MRH}$ compression method and $0.0046 \mathrm{~s}$ is attained by the standard MMR compression method. An average DPT minimization of $29.37 \%$ is attained for MRH over the standard MMR compression method.

An average OPT of $0.096 \mathrm{~s}$ is achieved by the proposed $\mathrm{MRH}$ and $0.0128 \mathrm{~s}$ is attained for the standard MMR compression method. An average OPT minimization of $24.56 \%$ is achieved by the proposed MRH compression method over the standard MMR compression method. The results attained demonstrates significant performance enhancement of proposed MRH compression method over the standard MMR compression method in terms of CE, EPT, DPT, OPT and CMU.

Table 2: Performance Evaluation of Encoding Processing Time, Decoding Processing Time and Overall Processing Time

\begin{tabular}{|c|c|c|c|c|c|c|}
\hline Images & \multicolumn{2}{|c|}{$\begin{array}{c}\text { EPT } \\
\text { (Secs) }\end{array}$} & \multicolumn{2}{c|}{$\begin{array}{c}\text { DPT } \\
\text { (Secs) }\end{array}$} & \multicolumn{2}{c|}{$\begin{array}{c}\text { OPT } \\
\text { (Secs) }\end{array}$} \\
\hline $\begin{array}{c}\text { Bi- } \\
\text { level }\end{array}$ & $\begin{array}{c}\text { Proposed } \\
\text { MRH }\end{array}$ & $\begin{array}{c}\text { Existing } \\
\text { MMR }\end{array}$ & $\begin{array}{c}\text { Proposed } \\
\text { MRH }\end{array}$ & $\begin{array}{c}\text { Existing } \\
\text { MMR }\end{array}$ & $\begin{array}{c}\text { Proposed } \\
\text { MRH }\end{array}$ & $\begin{array}{c}\text { Existing } \\
\text { MMR }\end{array}$ \\
\hline 1 & 0.055900 & 0.070836 & 0.003700 & 0.005154 & 0.092903 & 0.075990 \\
\hline 2 & 0.048311 & 0.091983 & 0.003200 & 0.005422 & 0.051511 & 0.097405 \\
\hline 3 & 0.084118 & 0.095432 & 0.003641 & 0.004268 & 0.087760 & 0.099700 \\
\hline 4 & 0.141076 & 0.231287 & 0.003285 & 0.004953 & 0.144361 & 0.236239 \\
\hline 5 & 0.085556 & 0.102907 & 0.003263 & 0.004614 & 0.090819 & 0.106521 \\
\hline 6 & 0.063926 & 0.079626 & 0.003193 & 0.004746 & 0.069120 & 0.083372 \\
\hline 7 & 0.164324 & 0.210008 & 0.003246 & 0.004740 & 0.168570 & 0.213748 \\
\hline 8 & 0.065258 & 0.108475 & 0.002999 & 0.003663 & 0.068257 & 0.112138 \\
\hline AVG & $\mathbf{0 . 0 8 8 5 5 9}$ & $\mathbf{0 . 1 2 3 8 1 9}$ & $\mathbf{0 . 0 0 3 3 1 6}$ & $\mathbf{0 . 0 0 4 6 9 5}$ & $\mathbf{0 . 0 9 6 6 6 3}$ & $\mathbf{0 . 1 2 8 1 3 9}$ \\
\hline
\end{tabular}

B. Performance evaluation considering bi-level images obtained from [16]:

Secondly, this work considers experimental analysis of the bi-level multimedia images obtained from [16]. The bi-level multimedia data considered for evaluation are shown in the Fig. 5 and has four images mentioned as 1,2,3,4. Images considered for experiment analysis possess identical dimension (Width*Height) of 256*256 with identical resolution of 72 dpi. The analysis is shown in the Fig.7. Where the results are compared with 7different algorithms. In the graph 1, 2, 3 and 4 indicates four images of Fig.6. From Fig.7, It can be seen that an average compression ratio performance for 7 cases are, LZW is 2.077, Deflate is 2.45, Packbits is 1.175, CCITT G3 is 1.725, CCITT G4 is 3.675, JBIG2 is 10.375 , ES [16] is 8.16, and for the Proposed MRH it is 13.28. The results attained shows that the proposed MRH model has achieved significant compression ratio performance improvement than the other state-of-the-art models mentioned above. An average CE enhancement of $35.25 \%$ is attained for presented MRH compression method over the state-of-the-art standard compression method of [16].

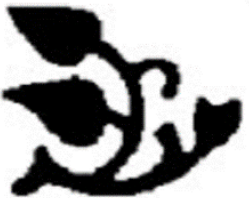

(1)

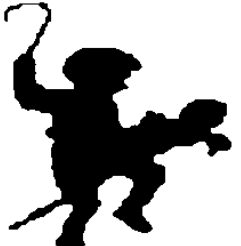

(3)

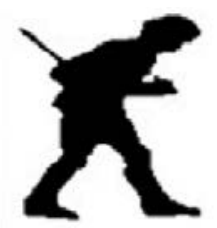

(2)

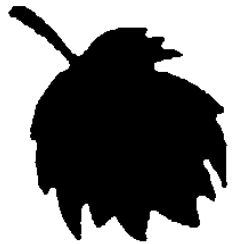

(4)
Fig. 6. Experiment result of MRH evaluated on bi-level images [16].

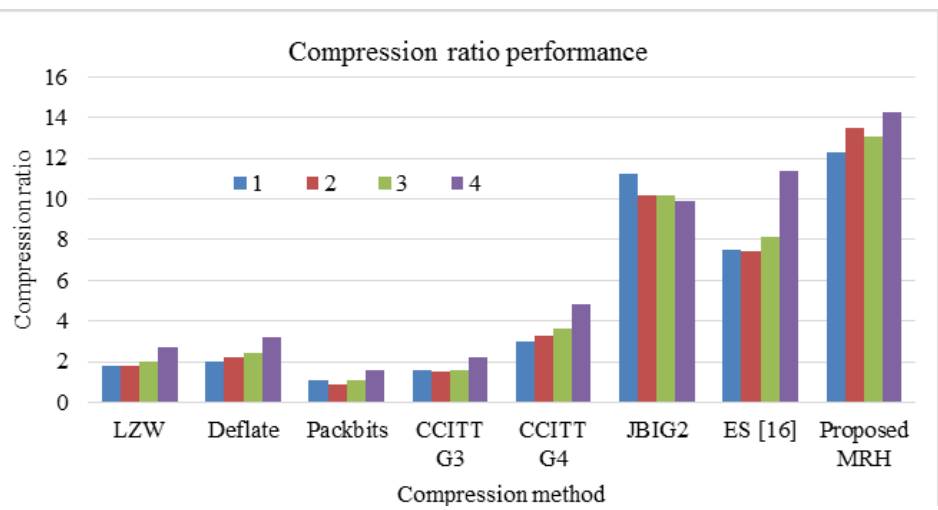

Fig. 7. Compression efficiency performance for bi-level images.

C. Performance evaluation by considering greyscale images:

Thirdly, this work considers the performance evaluation for greyscale images for the proposed MRH over the standard MMR compression method in terms of CE, EPT, DPT and CMU. The grey scale images used for analysis are obtained from [17, 25], 4 medical images in healthcare domain, 1 finger 
print image used for adoption of security purpose and 3 images are considered for general purpose analysis which are shown in the Fig.8. The grayscale images are first converted in 8-bit planes (binary images) are as shown in the Fig.9 for one image. These binary images are uncompressed individually. Encoding is performed individually on each bit plane using MRH to get compressed image. At the decoding stage, decoding is carried exactly in reverse manner of encoding and finally decoded planes are reconstructed into single grey scale image. All images have same resolution of 200 dpi and identical dimension of (Width*Height) of $1728 * 2339$.

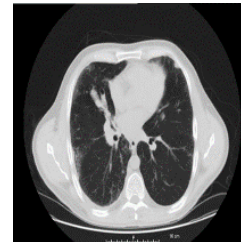

(1)

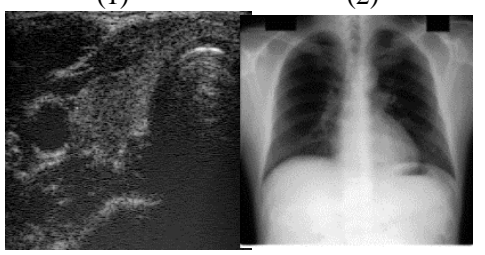

(3)

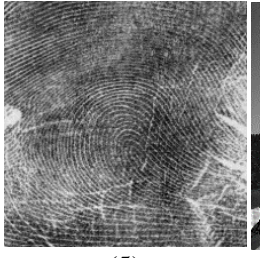

(5)

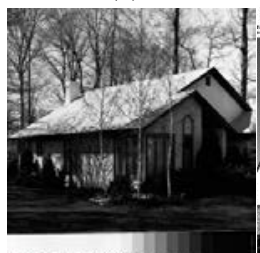

(7)

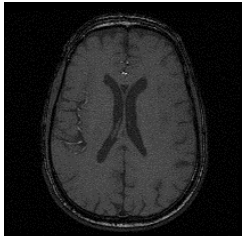

(2)

(4)

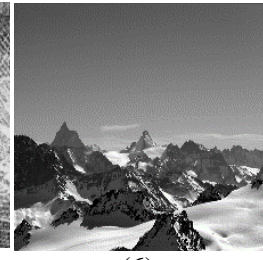

(6)

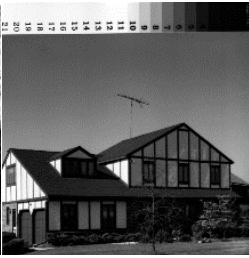

(8)
Fig. 8. Input greyscale images considered for Experimental analysis

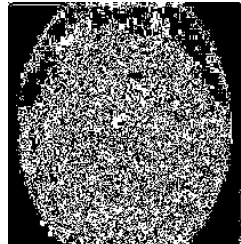

(1)

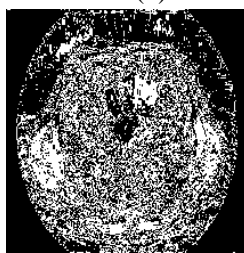

(3)

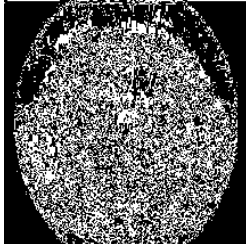

(2)

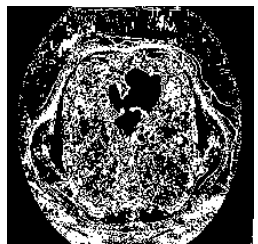

(4)

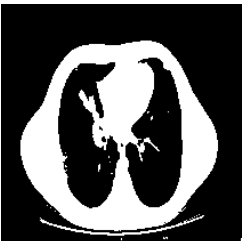

(5)

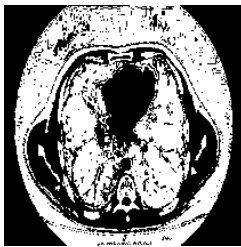

(7)

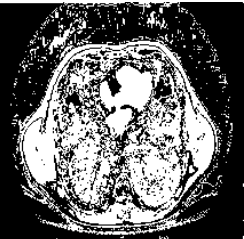

(6)

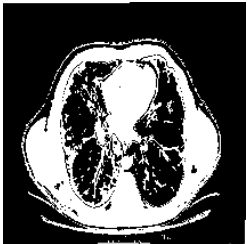

(8)
Fig.9. Bit Plane image slices for case 2 of figure 8 for the first image

The results are tabulated in Tables 3 and 4. The results attained shows that the proposed MRH compression method attained good enhancement over the standard MMR compression method in terms of CE, CMU and Overall Processing Time (OPT). The image 7 has attained highest compression ratio of 8.44 and image 5 has attained least compression ratio of 1.766. An average CE enhancement of $26.12 \%$ is attained by the proposed MRH compression method over the standard MMR technique. The proposed MRH utilize minimal memory compared with the standard MMR compression method. An average memory usage minimization of $0.855 \%$ is attained by the proposed MRH compression method over the standard MMR technique.

Table 3: Performance Evaluation of Compression Efficiency and Compression Memory Utilization

\begin{tabular}{|l|l|l|l|l|}
\hline Images & \multicolumn{2}{|l|}{ Compression Efficiency } & \multicolumn{1}{|c|}{$\begin{array}{l}\text { Compression } \\
\text { Memory Utilized (bytes) }\end{array}$} \\
\hline $\begin{array}{l}\text { Grey } \\
\text { scale }\end{array}$ & $\begin{array}{l}\text { Proposed } \\
\text { MRH }\end{array}$ & $\begin{array}{l}\text { Existing } \\
\text { MMR }\end{array}$ & $\begin{array}{l}\text { Proposed } \\
\text { MRH }\end{array}$ & $\begin{array}{l}\text { Existing } \\
\text { MMR }\end{array}$ \\
\hline 1 & 5.20003917 & 4.32704463 & 470840320 & 481044480 \\
\hline 2 & 6.74430279 & 5.48884566 & 471483904 & 471900672 \\
\hline 3 & 3.97636152 & 1.80069617 & 471150080 & 467564836 \\
\hline 4 & 4.15974388 & 3.41584634 & 470495232 & 466710016 \\
\hline 5 & 1.76633518 & 1.49408582 & 470983680 & 480972032 \\
\hline 6 & 3.38835620 & 2.85985586 & 471059456 & 469613696 \\
\hline 7 & 8.44619462 & 7.01375034 & 471300096 & 473582464 \\
\hline 8 & 6.28980533 & 5.29579735 & 471182848 & 489611904 \\
\hline AVG & 4.99639234 & 3.96199027 & 471061952 & 475125012 \\
\hline \multicolumn{2}{|r|}{} & & \\
\hline
\end{tabular}

Similarly, the MRH reduces OPT compared to the existing MMR compression method. The computation time performance is computed considering EPT, DPT, and OPT. An average EPT of 0.09s, an average DT of 0.0044s and an average OPT of $0.094 \mathrm{~s}$ is attained by the proposed MRH compression method. An average EPT of $0.102 \mathrm{~s}$, an average DT 0.0077sand an average OPT0.11s is attained for the standard MMR method shown in Table 4.

An average ET minimization of $11.76 \%$, an average DPT minimization of $42.8 \%$ and an average OPT time minimization of $14.54 \%$ is attained for proposed MRH compression method over the standard MMR compression method. The result obtained demonstrates enhancement of presented MRH 
compression method over the standard MMR compression method in terms of CE, EPT, DPT, OPT, and CMU.

Table 4: Performance Evaluation of Encoding processing time, Decoding processing time and Overall processing time

\begin{tabular}{|c|c|c|c|c|c|c|}
\hline Images & \multicolumn{2}{|c|}{$\begin{array}{c}\text { EPT } \\
\text { (Secs) }\end{array}$} & \multicolumn{2}{c|}{$\begin{array}{c}\text { DPT } \\
\text { (Secs) }\end{array}$} & \multicolumn{2}{c|}{$\begin{array}{c}\text { OPT } \\
\text { (Secs) }\end{array}$} \\
\hline $\begin{array}{c}\text { Grey } \\
\text { scale }\end{array}$ & $\begin{array}{c}\text { Proposed } \\
\text { MRH }\end{array}$ & $\begin{array}{l}\text { Existing } \\
\text { MMR }\end{array}$ & $\begin{array}{c}\text { Proposed } \\
\text { MRH }\end{array}$ & $\begin{array}{c}\text { Existing } \\
\text { MMR }\end{array}$ & $\begin{array}{c}\text { Proposed } \\
\text { MRH }\end{array}$ & $\begin{array}{l}\text { Existing } \\
\text { MMR }\end{array}$ \\
\hline 1 & 0.064886 & 0.066709 & 0.004188 & 0.037532 & 0.069074 & 0.104241 \\
\hline 2 & 0.060936 & 0.063913 & 0.004554 & 0.003437 & 0.065490 & 0.067350 \\
\hline 3 & 0.061155 & 0.053824 & 0.004268 & 0.003481 & 0.065423 & 0.057305 \\
\hline 4 & 0.010043 & 0.016759 & 0.004438 & 0.003582 & 0.014481 & 0.020341 \\
\hline 5 & 0.088905 & 0.070336 & 0.004008 & 0.003409 & 0.092913 & 0.073744 \\
\hline 6 & 0.135104 & 0.240620 & 0.004347 & 0.003534 & 0.139451 & 0.244154 \\
\hline 7 & 0.090044 & 0.098004 & 0.004031 & 0.003619 & 0.094075 & 0.101623 \\
\hline 8 & 0.209413 & 0.210595 & 0.004399 & 0.003204 & 0.213812 & 0.213799 \\
\hline AVG & $\mathbf{0 . 0 9 0 0 6 1}$ & $\mathbf{0 . 1 0 2 5 9 5}$ & $\mathbf{0 . 0 0 4 2 7 9}$ & $\mathbf{0 . 0 0 7 7 2 5}$ & $\mathbf{0 . 0 9 4 3 4 0}$ & $\mathbf{0 . 1 1 0 3 2 0}$ \\
\hline
\end{tabular}

\section{CONCLUSION}

The increasing demand for remote online health care services across various sectors and users has resulted in the increased sharing and storing of healthcare/medical diagnosis data. The cost per bit of storing data on cloud platform has come down which resulted in increased adoption of cloud based health care services. Minimizing cost per bit on such platform is most desired. Compression is an efficient mechanism for minimizing cost on such platform. The medical images stored on cloud platform are accessed by resource starved heterogeneous platform such as smart phones, smart devices etc. Therefore, minimizing the memory and computation time is most desired along with good lossless compression performance.

This paper presented an efficient lossless compression named MRH for greyscale (medical) images. The model presented overcomes the limitations of few of the state-of-theart techniques by adopting diagonal encoding and parallel computing for compressing an image. Experiments are conducted for wide variety of images and performance of the MRH is evaluated in terms of CE, OPT, and MU over the other state-of-the-art techniques, such as, LZW, Deflate, Packbits, CCITT G3, CCITT G4, JBIG2, MMR and LM back propagation algorithm.

MRH achieved good compression ratio performance over the existing MMR. An average compression efficiency enhancement of $17.29 \%$ and $26.12 \%$ is achieved by the MRH over the MMR considering bi-level document images and greyscale images respectively. For another case, results are compared with LM back propagation algorithm which is a Neural Network based compression algorithm. An average CE enhancement of $35.25 \%$ is achieved by MRH over Neural network based compression algorithm considering bi-level images. The MRH attains good performance improvement in terms of EPT, DPT, and OPT and CMU over its stat-of-the-art technique.
An average improvement of 28.37\%, 29.37\% and 24.56\% and $2.07 \%$ is achieved by the MRH compression method over the standard MMR Method, respectively for bi-level document images. An average enhancement of $26.12 \%, 0.855 \%, 11.76 \%$, 42.8\%, $14.54 \%$ are achieved by the MRH compression method over the standard MMR compression method, respectively, for greyscale images. The overall outcome achieved shows that, the proposed MRH is a good scalable, efficient and robust performance compression technique and applicability of MRH for different application services.

In future work, we would consider implementation of RH and MRH compression techniques as an efficient Fronthaul compression technique for future generation wireless communication network such as C-RAN. An attempt will be made to increase the bit rate, speed of transmission and reduce the bandwidth using the proposed MRH compression technique.

\section{REFERENCES}

[1] Schlupkothen, F.R.N., "Interoperability between medical image archives and consumer devices through web services," Consumer Electronics (ICCE), 2012 IEEE International Conference on , vol., no., pp.480,481, 13-16 Jan. 2012.

[2] NEMA PS3 / ISO 12052, Digital Imaging and Communications in Medicine (DICOM) Standard, National Electrical Manufacturers Association, Rosslyn, VA, USA (available free at http://medical.nema.org/), last access on 22, october 2017.

[3] Singara Singh , R. K. Sharma, M.K. Sharma, "Use of Wavelet Transform Extension for Graphics Image Compression using JPEG2000 Framework”, International Journal of Image Processing, Volume 3, Issue 1, Pages 55-60, 2009.

[4] Steven Pigeon, Yoshua Bengio,"A Memory-Efficient Huffman Adaptive Coding Algorithm for Very Large Sets of Symbols Revisited “, Université de Montréal, Rapport technique \#1095.

[5] Chiu-Yi Chen; Yu-Ting Pai; Shanq-Jang Ruan, Low Power Huffman Coding for High Performance Data Transmission, International Conference on Hybrid Information Technology, 2006, 1(9-11), 2006 pp. $71-77$.

[6] R. Ponalagusamy and C. Saravanan, Analysis of Medical Image Compression using Statistical Coding Methods, Advances in Computer Science and Engineering: Reports and Monographs, Imperial College Press, UK, Vol.2., pp 372-376, 2007.

[7] S. Parikh, H. Kalva and V. Adzic, "Evaluation of HEVC compression for high bit depth medical images," IEEE International Conference on Consumer Electronics (ICCE), Las Vegas, NV, pp. 311-314, 2016.

[8] S. Parikh; D. Ruiz; H. Kalva; G. Fernandez-Escribano; v. Adzic, "High Bit-Depth Medical Image Compression with HEVC," in IEEE Journal of Biomedical and Health Informatics, vol.PP, no.99, pp.1-1, 2017.

[9] G. J. Sullivan, J. R. Ohm, W. J. Han and T. Wiegand. Overview of the High Efficiency Video Coding (HEVC) Standard. IEEE Transactions on Circuits and Systems for Video Technology. 22(12), pp. 1649-1668, 2012.

[10] P. Enfedaque; F. Auli-Llinas; J. C. Moure, "GPU Implementation of Bitplane Coding with Parallel Coefficient Processing for High Performance Image Compression," in IEEE Transactions on Parallel and Distributed Systems , vol.PP, no.99, pp.1-1. 2017.

[11] F. Aulí-Llinàs, "Context-Adaptive Binary Arithmetic Coding With Fixed-Length Codewords," in IEEE Transactions on Multimedia, vol. 17, no. 8, pp. 1385-1390, 2015.

[12] A. Zribi, R. Pyndiah, S. Zaibi, F. Guilloud and A. Bouallegue, "LowComplexity Soft Decoding of Huffman Codes and Iterative Joint Source 
Channel Decoding," in IEEE Transactions on Communications, vol. 60, no. 6, pp. 1669-1679, 2012.

[13] H. C. Kuo and Y. L. Lin, "A Hybrid Algorithm for Effective Lossless Compression of Video Display Frames," in IEEE Transactions on Multimedia, vol. 14, no. 3, pp. 500-509, 2012.

[14] D. A. Huffman, "A method for the construction of minimum redundancy codes,” Proc. I.R.E., vol. 40, no. 9, pp. 1098-1102, Sep. 1952.

[15] Andreas E. Savakis,”Evaluation Of Lossless Compression Methods for Gray Scale Document Images”,2000 IEEE, pp.no 136-139.

[16] S. Sahami M.G. Shayesteh, "Bi-level image compression technique using neural networks,” IET Image Process., Vol. 6, Iss. 5, pp. 496-506, 2012.

[17] T. Kavitha and Dr. K. Jaya Sankar, "An Efficient Compression Technique for ITU-T Group 3 Coded Images Using Variable Length Codes with Reduced Average Length”, 2016 IEEE International Conference on India International Conference On Information Processing (IICIP-2016) , pp. 1-6.

[18] "//www.itu.int/net/itu-t/sigdb/genimage/test24.htm".

[19] K. Khursheed, N. Ahmad, M. Imran and M. O'Nils, "Detecting and coding region of interests in bi-level images for data reduction in Wireless Visual Sensor Network," 2012 IEEE 8th International Conference on Wireless and Mobile Computing, Networking and Communications (WiMob), Barcelona, 2012, pp. 705-712, 2012.

[20] A. Niemi and J. Teuhola, "Interpolative Coding as an Alternative to Arithmetic Coding in Bi-Level Image Compression," SCC 2015; 10th International ITG Conference on Systems, Communications and Coding, Hamburg, Germany, pp. 1-6, 2015.

[21] Fahad Lateef, Najeem Lawal, Muhammad Imran "Binary Image Compression Algorithms for FPGA Implementation,” International Journal of Scientific \& Engineering Research, Volume 7, Issue 3, March-2016.

[22] T. Kavitha and Dr. K. Jaya Sankar, "Ideal Huffman Code for Efficient Lossless Image Compression for Ubiquitous Access", Indonesian Journal of Electrical Engineering and Computer ,p-ISSN: 2502-4752, eISSN: 2502-4760.

[23] Arun.N.Netravali,Frank W.Mounts," Ordering Techniques for Facsimile Coding: A Review”, Proceedings Of The IEEE 1980, Vol. 68, No. 7, July 1980. pp. No.796-807.

[24] Yasuhiko Yasuda,” Overview of Digital Facsimile Coding Techniques in Japan”,Proceedings Of The IEEE, Vol. 68, No. 7, July 1980,pp.No.830-845.

[25] https://www.aycan.de/sample-dicom-images.html.

[26] Ki-Hyun Jung, Comparative Histogram Analysis of LSB-based Image Steganography, WSEAS Transactions on Systems and Control, Volume 13, 2018, pp. 103-112.

[27] Lakshmi K, Robert Theivadas J and Markkandan, ,Variable-to-Variable Run Length Encoding Technique for Testing Low Power VLSI Circuits”, S, Journal of Electrical and Electronic Systems 2019, 8:1 DOI: $10.4172 / 2332-0796.1000300$.

[28] P. Enfedaque; F. Auli-Llinas; J. C. Moure, "GPU Implementation of Bitplane Coding with Parallel Coefficient Processing for High Performance Image Compression," in IEEE Transactions on Parallel and Distributed Systems, vol.PP, no.99, pp.1-1. 2017.

[29] X. Ma, Z. Pan, Y. Li and J. Fang, "High-quality initial codebook design method of vector quantisation using grouping strategy," in IET Image Processing, vol. 9, no. 11, pp. 986-992, 2015.

[30] F. Aulí-Llinàs, "Context-Adaptive Binary Arithmetic Coding With Fixed-Length Codewords," in IEEE Transactions on Multimedia, vol. 17, no. 8, pp. 1385-1390, 2015.

[31] Yih-Chuan Lin and Shen-Chuan Tai, "A fast Linde-Buzo-Gray algorithm in image vector quantization," in IEEE Transactions on
Circuits and Systems II: Analog and Digital Signal Processing, vol. 45, no. 3, pp. 432-435, March 1998.

[32] L. Gang, L. Jing and W. Quan, "A Robust Lin-Buzo-Gray Algorithm in Data Vector Quantization," 2009 International Forum on Information Technology and Applications, Chengdu, 2009, pp. 464-467.

[33] A. Skodras, C. Christopoulos and T. Ebrahimi, "The JPEG 2000 still image compression standard," in IEEE Signal Processing Magazine, vol. 18, no. 5, pp. 36-58, Sept. 2001. 